\title{
TRIANGLE DECOMPOSITIONS OF PLANAR GRAPHS
}

\author{
Christina M. Mynhardt ${ }^{1}$
}

AND

\author{
Christopher M. van BommeL ${ }^{2,3}$ \\ Department of Mathematics and Statistics \\ University of Victoria \\ P.O. Box 1700 STN CSC \\ Victoria, BC, Canada V8W $2 Y 2$
}

e-mail: kieka@uvic.ca, cvanbomm@uwaterloo.ca

\begin{abstract}
A multigraph $G$ is triangle decomposable if its edge set can be partitioned into subsets, each of which induces a triangle of $G$, and rationally triangle decomposable if its triangles can be assigned rational weights such that for each edge $e$ of $G$, the sum of the weights of the triangles that contain $e$ equals 1 .

We present a necessary and sufficient condition for a planar multigraph to be triangle decomposable. We also show that if a simple planar graph is rationally triangle decomposable, then it has such a decomposition using only weights 0,1 and $\frac{1}{2}$. This result provides a characterization of rationally triangle decomposable simple planar graphs. Finally, if $G$ is a multigraph with $K_{4}$ as underlying graph, we give necessary and sufficient conditions on the multiplicities of its edges for $G$ to be triangle and rationally triangle decomposable.
\end{abstract}

Keywords: planar graphs, triangle decompositions, rational triangle decompositions.

2010 Mathematics Subject Classification: 05C10, 05C70.

\footnotetext{
${ }^{1}$ Supported by a Discovery Grant from the Natural Sciences and Engineering Research Council of Canada.

${ }^{2}$ Supported by a Julie Payette Research Scholarship and an André Hamer Postgraduate Prize from the Natural Sciences and Engineering Research Council of Canada.

${ }^{3}$ Currently PhD student, Department of Combinatorics and Optimization, University of Waterloo, Waterloo, ON, Canada.
} 


\section{REFERENCES}

[1] B. Barber, D. Kühn, A. Lo and D. Osthus, Edge-decompositions of graphs with high minimum degree, arXiv:1410.5750v3, 2015. 
[2] A. Bialostocki and Y. Roditty, $3 K_{2}$-decomposition of a graph, Acta Math. Acad. Sci. Hungar. 40 (1982) 201-208. doi:10.1007/BF01903577

[3] N.L. Biggs, T.P. Kirkman, Mathematician, Bull. Lond. Math. Soc. 13 (1981) $97-120$. doi:10.1112/blms/13.2.97

[4] O. Borodin, A.O. Ivanova, A. Kostochka and N.N. Sheikh, Planar graphs decomposable into a forest and a matching, Discrete Math. 309 (2009) 277-279. doi:10.1016/j.disc.2007.12.104

[5] F. Dross, Fractional triangle decompositions in graphs with large minimum degree, arXiv:1503.08191v3, 2015.

[6] O. Favaron, Z. Lonc and M. Truszczyński, Decompositions of graphs into graphs with three edges, Ars Combin. 20 (1985) 125-146.

[7] K. Garaschuk, Linear Methods for Rational Triangle Decompositions, Doctoral Dissertation (University of Victoria, 2014). http://hdl.handle.net/1828/5665

[8] Z. Lonc, M. Meszka and Z. Skupień, Edge decompositions of multigraphs into 3matchings, Graphs Combin. 20 (2004) 507-515. doi:10.1007/s00373-004-0581-0

[9] R. Häggkvist and R. Johansson, A note on edge-decompositions of planar graphs, Discrete Math. 283 (2004) 263-266. doi:10.1016/j.disc.2003.11.017

[10] I. Holyer, The NP-completeness of some edge-partition problems, SIAM J. Comput. 10 (1981) 713-717. doi:10.1137/0210054

[11] P. Keevash, The existence of designs, arXiv:1401.3665v1, 2014.

[12] S.-J. Kim, A.V. Kostochka, D.B. West, H. Wu and X. Zhu, Decomposition of sparse graphs into forests and a graph with bounded degree, J. Graph Theory 74 (2013) 369-391.

doi:10.1002/jgt.21711

[13] T. Kirkman, On a problem in combinations, The Cambridge and Dublin Mathematical Journal (Macmillan, Barclay, and Macmillan) II (1847) 191-204.

[14] C.St.J.A. Nash-Williams, An unsolved problem concerning decomposition of graphs into triangles, in: P. Erds, P. Rnyi and V.T. Sós (Eds.), Combinatorial Theory and its Applications III (North Holland, 1970) 1179-1183.

[15] J. Steiner, Combinatorische Aufgaben, J. Reine Angew. Math. 45 (1853) 181-182. doi:10.1515/crll.1853.45.181

[16] Y. Wang and Q. Zhang, Decomposing a planar graph with girth at least 8 into a forest and a matching, Discrete Math. 311 (2011) 844-849.

doi:10.1016/j.disc.2011.01.019 
[17] D.B. West, Introduction to Graph Theory (Prentice Hall, Inc., Upper Saddle River, NJ, 1996).

[18] W.S.B. Woolhouse, Prize question \#1733, Lady's and Gentleman's Diary (1844) London, Company of Stationers.

Received 15 April 2015

Revised 6 October 2015

Accepted 6 October 2015 